\section{Vom Symptom zur Diagnose}

— Im Spätdienst nimmt der junge Assistenzarzt vor Dienstschluss noch schnell ein Kind mit Fazialisparese auf. Nach der Blutund Liquorpunktion, den üblichen serologischen Tests auf Borrelien und dem Anlegen einer Kultur ist die Routinediagnostik zunächst einmal beendet. Doch was, wenn sich keine wegweisenden Befunde ergeben? Welche anderen Differenzialdiagnosen kommen in Betracht? Gäbe es weitere diagnostische Maßnahmen, die der Pädiater ergreifen sollte?

Mit der ersten Auflage des Buches „Differenzialdiagnosen in der Kinder- und Jugendmedizin" hat Robert Ploier ein Werk geschaffen, welches genau an dieser Stelle im klinischen Alltag seine Anwendung finden kann. Alphabetisch geordnet, lassen sich wichtige Symptome nachschlagen. Dabei stößt der Routinier gelegentlich auch auf Diagnosen oder Zusammenhänge, die ihm

\section{Migräne in jedem Alter}

— Die Volkskrankheit Migräne tritt bereits im Kleinkindesalter bei rund 3,2\% aller Kinder auf und steigert sich bis zum Schulalter auf ca. 12\%. Rechtzeitiges Erkennen mindert den Leidensdruck des betroffenen Kindes und erspart ihm eventuell überflüssige Diagnostik. Der Phänotyp der Migräne im Jugendalter deckt sich weitgehend mit dem der Erwachsenen. Im Kindesalter variiert er jedoch erheblich.

Auf 360 Seiten stellt der Autor des Werks "Migräne", Leiter des Migräne- und Kopfschmerzzentrums in Kiel, aktuelles und umfangreiches Wissen über die Erkrankung vor. Im ersten Kapitel geht er auf die sozioökonomischen Folgen und auf die Defizite der Versorgung ein. Das zweite Kapitel ist der IHS-Klassifikation gewidmet.

Mithilfe von Fallbeispielen wird die Phänotypologie der Migräne und ihrer Varianten beschrieben. Geschichte, Genetik, Pathophysiologie, Triggerfaktoren und Differen- nicht mehr unbedingt geläufig sind. Auch pathologische Laborwerte des Patienten können in diesem Buch nachgeschlagen werden. Beispielsweise finden sich unter dem Schlagwort "Hypoglykämie" nicht nur die Differenzialdiagnosen, sondern auch eine Tabelle zu altersentsprechenden Blutzuckergrenzwerten sowie wichtige klinische Anmerkungen des Autors in "Merke"Kästchen. Bei diesen Anmerkungen handelt es sich oft um Tipps aus dem klinischen Alltag bzw. kurze Auszüge aus Leitlinien. Insgesamt ist dieses Werk ein sehr praxisorientiertes Buch, das keinen Anspruch auf komplette Vollständigkeit erhebt (hierauf weist der Autor auch in seinem Vorwort ausdrücklich hin). Es stellt ein effektives Nachschlagewerk dar und trägt zur schnelleren Diagnosefindung im klinischen Alltag bei. Leider wirken die zweifarbig gehaltenen Seiten durch die vielen Kästchen und

zialdiagnosen werden detailliert dargelegt. Auch Verlauf und Prognose werden erörtert. Therapeutische Möglichkeiten, von alternativen Maßnahmen über die klassische medikamentöse Migränetherapie bis hin zur Botoxtherapie, werden im Anschluss vorgestellt.

Der Migräne im Kindesalter sind 20 Seiten gewidmet. Der Autor geht auf die Abweichungen in der Symptomatik und auf die im Kindesalter möglichen Migräneäquivalente ein. Er diskutiert die - aufgrund der anamnestisch schwierig zu erhebenden Informationen - diagnostischen und differenzialdiagnostischen Konsequenzen. Hinsichtlich der medikamentösen Therapie ergeben sich deutliche Unterschiede zu den Erwachsenen, die vom Autor übersichtlich aufgezeigt werden.

Die klinische Erfahrung des Autors ermöglicht Praxistipps und konkrete Handlungsanweisungen. Wichtige Informationen sind
Tabellen teilweise unübersichtlich und es fehlt dem geneigten Leser manchmal ein einführender Text. Wer sich jedoch mit der spartanischen Schreibweise in Stichwörtern arrangieren kann, dem steht - egal ob in Klinik oder Praxis - ein hilfreiches Buch zur Verfügung, das auf 419 Seiten viel Wissen in komprimierter Form darbietet. Leider ist das Preis-Leistungs-Verhältnis noch etwas ungünstig, was sicherlich der geringen Auflagenzahl geschuldet ist. Wie wäre es mit einer Ausgabe als eBook für das Smartphone?

Jessica Bockhorst

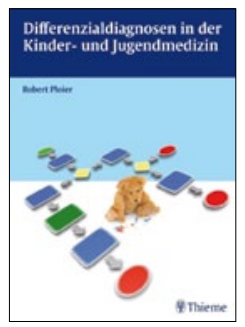

Robert Ploier

Differenzialdiagnosen in der Kinder- und Jungendmedizin

Georg Thieme Verlag, Stuttgart 2013

$149,99 €$

ISBN 978-3-13-171161-8

mit blauer Farbe unterlegt, sodass es dem Leser in kurzer Zeit möglich ist, die essenziellen Fakten zu erfahren. Mit etwas mehr Zeit wird er den gesamten Text lesen können, der auch im müden Zustand gut lesbar ist und mehr Verständnis für die auch im Kindesalter so häufig auftretende Erkrankung vermittelt.

Das Buch ist nicht nur den selbst von Migräne betroffenen Kollegen, sondern auch im Hinblick auf seine kleinen Patienten sehr zu empfehlen.

Dr. Kirsten Stollhoff

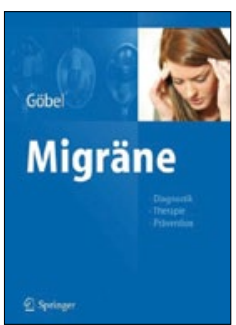

Hartmut Göbel

Migräne

Springer-Verlag, Heidelberg 2012

$69,95 €$

ISBN 3-642-25557-4 